\title{
Centroamérica joven: valores democráticos en la generación de la posguerra
}

Florisabel Rodríguez y Silvia Castro Méndez ${ }^{1}$

El presente artículo busca generar insumos para todos aquellos sectores sociales interesados en el entendimiento y la promoción de la cultura democrática en Centroamérica. Se centra en un tema fundamental de la cultura política: el estudio de los valores de la igualdad política, la solidaridad y la tolerancia hacia la diversidad.

La presencia de estos valores en la ciudadanía no es condición sine qua non de la democracia como régimen político. No obstante, como lo ha planteado la teoría normativa de la democracia, dicha presencia incrementa las oportunidades para profundizar la democracia, mejorar su calidad y sostenibilidad.

1. Florisabel Rodríguez. Polítologa costarticense. Exministra de información del gobiemo de Costa Rica. Directora y fundadora de Procesos. E-mail: florisabel@procesos.org Silvia Castro Méndez. Filossofa y poeta costarricense. Consultora en temas de transferencia tecnológica, comunicación política y cultura.E-mail: scastro@procesos.org 
La investigación que nutre este artículo se basa en una amplia encuesta autoadministrada aplicada a estudiantes de sétimo y undécimo año de secundaria de colegios diurnos públicos y privados, en las áreas metropolitanas de los países centroamericanos, con la excepción de Belice y Panamá ${ }^{2}$. Se escogieron estos dos niveles educativos para tratar de identificar diferencias entre unos y otros, y obtener así mayor claridad con respecto a los procesos de formación y consolidación de sus valores en estos años.

Para seleccionar la muestra de estudiantes se utilizó la técnica de probabilidad proporcional al tamaño (PPT), definida según información de matrícula de sétimo y undécimo año, o general del colegio, según se tuvo acceso en los países. Se seleccionó el número de colegios necesario para obtener alrededor de 750 entrevistas en cada nivel ${ }^{3}$. El trabajo de campo fue realizado fundamentalmente en el año de $2000^{4}$ y en total se entrevistaron 8433 estudiantes de sétimo y de undécimo año.

El análisis incluye comparaciones por país, por nivel educativo y por género. A este último se hará referencia solo cuando existan diferencias relevantes.

\section{Algunas apreciaciones sobre los valores democráticos}

\section{La igualdad política}

Una de las características fundamentales de la democracia es el derecho que tienen todos y todas las ciudadanas de emitir un voto único, cuyo peso específico es idéntico al de cualquier otro ciudadano, no importa su condición

\footnotetext{
2. En el caso de Honduras, se incluyeron Tegucigalpa y San Pedro Sula. En el caso de Costa Rica se incluyeron, además de San José, las tres capitales de provincia que se encuentran en la Gran Área Metropolitana: Alajuela, Heredia y Cartago.

3. Agradecemos a Miguel Gómez Barrantes haber realizado el diseño muestral.

4. Solamente la encuesta de Costa Rica fue realizada en 1998.
} 
particular. Esta característica central del sistema político democrático es una profunda expresión del valor de la igualdad política: todas y todos los ciudadanos tienen un conjunto inalienable de derechos.

El valor de la igualdad política puede trascender las convicciones puramente individuales y adquirir una dimensión institucional con el establecimiento de una serie de normas, leyes y procedimientos, que buscan extender la igualdad formal en lo público y minimizar las formas de la arbitrariedad.

La correspondencia que se haya formado en cada democracia específica entre los valores de los individuos y las normas e instituciones del sistema político fortalece o desgasta la cultura democrática. De manera que, si el valor de la igualdad es ampliamente compartido por el conjunto de los ciudadanos y éste se ve retratado también institucionalmente, entonces se espera que el apoyo al sistema tienda a verse fortalecido y haya un reconocimiento colectivo a la virtud que encierran los lazos de reciprocidad, respeto y cooperación por encima de las formas de la dependencia, la arbitrariedad y del autoritarismo (Putnam, 1993).

Del valor de la igualdad se derivan filosóficamente los valores de la tolerancia hacia la diversidad y de la solidaridad.

\section{La tolerancia hacia la diversidad}

La tolerancia hacia quienes son diferentes es una forma específica en la que se manifiesta el valor de la igualdad. Afirma los derechos de las personas con las que se difiere o se disiente, ya sea por razones de pertenencia étnica, posición ideológica, creencia religiosa, género, condición social o económica, preferencias políticas, preferencias sexuales, etc. En otras palabras, es un valor que permite la coexistencia 
pacífica y respetuosa de personas o grupos de personas con distintas historias, formas de pensar, culturas e identidades (Walzer, 1997).

La aceptación de la diversidad puede ser indiferente, pasiva, estoica o entusiasta; lo cierto es que todo aquel capaz de dar el salto desde sus creencias para dar cabida al derecho de quien es distinto puede ser llamado una persona tolerante.

En el ámbito político la tolerancia hacia la diversidad favorece el respeto entre minorías y mayorías, la aceptación del derecho al desacuerdo, y la igualdad de derechos entre quienes son diferentes.

\section{La solidaridad}

El valor de la solidaridad es la extensión del valor de la igualdad hacia el ámbito de lo social y lo económico, al afirmar la necesidad de crear oportunidades para el bienestar de toda la ciudadanía.

Este valor puede tener manifestaciones altruistas, con exigencias orientadas hacia el bienestar de los demás. Pero también puede ser entendido como una exigencia básica para alcanzar el propio bienestar ${ }^{5}$. Los espacios para las expresiones de solidaridad pueden ser individuales o colectivos, públicos o privados.

Cualquiera sea la manera en la cual se ejerza la solidaridad, es un valor que tiende a mejorar el tejido social y generar la demanda hacia el sistema político por mayores oportunidades para los que menos tienen. La importancia

\footnotetext{
5. En este último sentido, el interés individual se asocia al colectivo en lo que Tocyueville (1969) llamó el interés individual adecuadamente entendido. De acuendo con esto, solo en una sociedad en que se busque minimizar las causas de la exclusión social y económica, y se logren formas más integradoras de crecimiento material para la población, es posible lograr el bienestar pleno de cada uno de los individuos particula:mente.
} 
para la sostenibilidad de las democracias de construir una mayor igualdad de oportunidades ha sido señalada por numerosos estudios ${ }^{6}$.

\section{Distribución de los valores}

\section{La igualdad política}

El valor de la igualdad se midió mediante tres preguntas. La primera se refiere al derecho al voto, la segunda al derecho a la defensa en un juicio, y la última, al derecho a pedir apoyo para defender una causa.

Para medir lo atinente al derecho al voto, la pregunta se refiere a la conveniencia de que exista ese derecho incluso para aquellos ciudadanos que no puedan ejercitarlo de manera inteligente?

El motivo para realizar una pregunta con estas características es presentar una situación aparentemente problemática asociada al derecho particular, para identificar así hasta qué punto las personas oponen resistencia al argumento antidemocrático.

La medición del valor de la igualdad en los estudiantes de ambos niveles educativos muestra un nivel alto, dado que en todos los países, un 70\% o más de los jóvenes están "muy de acuerdo" o "de acuerdo" con la afirmación de que todas las personas tienen derecho al voto, independientemente de la calidad con que se ejerza su decisión (Cuadro 1). Es notable el caso de Costa Rica, país en el cual un $85 \%$ de los estudiantes de undécimo manifestó estar "muy de acuerdo" o "de acuerdo" con esa posición ${ }^{8}$.

6. Uno de los estudios clásicos que lo señala es el de Seymour M. Lipset (1963).

7. La pregunta dice: "A toda la gente se le debe permitir votar aunque no lo haga inteligentemente ¿Está usted de acuerdo o en desacuerdo?".

8. Esta masiva aceptación del derecho al voto en Costa Rica puede estar obedeciendo a la continuidad de la experiencia democrática vivida en materia electoral durante las últimas cinco décadas. La legitimidad del voto a partir de esa experiencia se ha 
Cuadro 1

\section{Derecho al voto aún cuando no se ejerza} inteligentemente por país según nivel de estudios (en porcentajes)

Nivel de estudios Guatemala Honduras El Salvador Nicaragua Costa Rica

\begin{tabular}{lrrrrr}
\hline Total & 100 & 100 & 100 & 100 & 100 \\
& & & & & \\
(N) & $(754)$ & $(1099)$ & $(809)$ & $(871)$ & $(562)$ \\
Undécimos & & & & & \\
$\quad$ Aprueba & 74.1 & 69.7 & 69.8 & 71.3 & 84.9 \\
Desaprueba & 24.4 & 27.7 & 28.0 & 25.7 & 13.9 \\
Ns/Nr & 1.5 & 2.6 & 2.3 & 3.0 & 1.2 \\
& & & & & \\
(N) & $(805)$ & $(1205)$ & $(783)$ & $(964)$ & $(581)$ \\
Sétimos & & & & & \\
Aprueba & 74.1 & 70.6 & 71.9 & 71.7 & 77.5 \\
Desaprueba & 23.5 & 26.2 & 25.6 & 22.7 & 19.1 \\
Ns/Nr & 2.4 & 3.2 & 2.6 & 5.6 & 3.4 \\
\hline
\end{tabular}

La segunda pregunta se refiere al derecho de cualquier persona a tener una defensa en un juicio, independientemente del delito que haya cometido ${ }^{9}$. Al mencionar la independencia del delito, es posible conocer hasta qué punto las y los encuestados están dispuestos a apoyar el principio democrático por encima de la valoración concreta sobre el crimen particular. Además, considerar un indicador de la igualdad ante la justicia es importante puesto que la administración igualitaria de la justicia es uno de los pilares del edificio democrático.

consolidado en la mentalidad de los costarricenses como uno de los pilares sobre los que reposa su democracia.

9. La pregunta dice: " ¿Cree usted que cualquier persona debe tener derecho a una buena defensa en un juicio en los Tribunales de Justicia, no importa el delito que haya cometido?". 
Las respuestas también dan resultados muy positivos sobre la distribución del valor de la igualdad (Cuadro 2). En todos los países, más de un $74 \%$ de los y las jóvenes de undécimo año están de acuerdo con que el derecho a la defensa judicial sea otorgado a todas las personas. Honduras es notable pues un $80 \%$ de los de undécimo lo expresan.

\section{Cuadro 2}

Derecho a la defensa judicial sin importar el delito por país según nivel de estudios

(en porcentajes)

Nivel de

estudios

Guatemala Honduras El Salvador Nicaragua Costa Rica

Total

100

100

100

100

100

(N)

(754) (1099)

(809)

(871)

(562)

Undécimos

$\begin{array}{lrrrrr}\text { Aprueba } & 77.1 & 80.2 & 74.7 & 79.0 & 74.0 \\ \text { Desaprueba } & 16.0 & 12.8 & 18.5 & 14.4 & 16.7 \\ \mathrm{Ns} / \mathrm{Nr} & 6.9 & 7.0 & 6.8 & 6.7 & 9.3\end{array}$

$(\mathrm{N})$

(805) (1205)

(783)

(964)

(581)

Sétimos

$\begin{array}{lrrrrr}\text { Aprueba } & 74.2 & 74.4 & 72.3 & 72.8 & 67.6 \\ \text { Desaprueba } & 16.1 & 18.6 & 17.5 & 19.5 & 20.7 \\ \mathrm{~N} s / \mathrm{Nr} & 9.7 & 7.0 & 10.2 & 7.7 & 11.7\end{array}$

La tercera pregunta es la que corresponde al derecho de las personas a solicitar apoyo para defender una causa en la que creen ${ }^{10}$ (Cuadro 3). Esta pregunta lleva implícito el derecho a la libre expresión y al de organización, derechos

10. La pregunta dice: "¿Cree usted que todas las personas en nuestro país deben tener igual derecho a pedir apoyo de otros para defender una causa en la que ellas creen?". 
básicos y universales en todo sistema democrático. No se incluyó ningún condicionante como en el caso de las preguntas anteriores y esto posiblemente afecta la respuesta afirmativa que supera el $90 \%$ en todos los casos ${ }^{11}$.

\section{Cuadro 3 \\ Derecho a solicitar apoyo para defender una causa por país según nivel de estudios (en porcentajes)}

\begin{tabular}{lccccc}
$\begin{array}{l}\text { Nivel de } \\
\text { estudios }\end{array}$ & Guatemala & Honduras & El Salvador & Nicaragua & Costa Rica \\
\hline Total & 100 & 100 & 100 & 100 & 100 \\
& & & & & \\
$(\mathrm{~N})$ & $(754)$ & $(1099)$ & $(809)$ & $(871)$ & $(562)$ \\
Undécimos & & & & & \\
$\quad$ Aprueba & 90.1 & 93.0 & 89.7 & 91.3 & 90.7 \\
$\quad$ Desaprueba & 6.5 & 4.4 & 6.2 & 4.4 & 6.0 \\
Ns/Nr & 3.4 & 2.6 & 4.1 & 4.4 & 3.2 \\
& & & & & \\
$(\mathrm{~N})$ & $(805)$ & $(1205)$ & $(783)$ & $(964)$ & $(581)$ \\
Sétimos & & & & & \\
Aprueba & 92.5 & 91.8 & 93.5 & 91.6 & 91.0 \\
$\quad$ Desaprueba & 5.6 & 6.2 & 4.3 & 5.5 & 6.5 \\
Ns/Nr & 1.9 & 2.0 & 2.2 & 2.9 & 2.4 \\
\hline
\end{tabular}

11. La fortaleza de la respuesta a favor de la igualdad podría ser, al mismo tiempo, una debilidad desde el punto de vista de la medición de ese valor, puesto que no se introdujo ningún elemento problemático o controversial y no se puede saber, for lo tanto, hasta qué punto las personas jóvenes consideran que el derecho a pedir apoyo por una causa determinada debe ser mantenido por encima de la situación particular (como sería la consideración del caso de quienes defienden regímenes autoritarios, o causas a favor de la pena de muerte, del aborto o de la eutanasia). 
En este estudio se utilizó el método que parte de identificar al grupo menos gustado por la o el entrevistado dentro de un conjunto de grupos que sufren formas de discriminación social o política (Booth y Seligson, 1993).

La tolerancia hacia la diversidad se midió por medio de cuatro preguntas: con la primera se logra determinar el grupo humano que menos le gusta al entrevistado o entrevistada. Las siguientes tres preguntas se refieren a derechos ciudadanos sobre los que se opina si deben o no serles concedidos a las personas pertenecientes al grupo previamente mencionado como el menos grato, cualquiera que éste haya sido $^{12}$ : enseñar en las escuelas públicas, ser electo o electa en cargos públicos y hablar en televisión y en reuniones públicas.

Se considera que los más tolerantes son aquellos a quienes ningún grupo de la sociedad les disgusta, o bien que, identificando uno, consideran que éste debe contar con los derechos mencionados.

La distribución porcentual del grupo menos gustado se presenta en el Cuadro 4. Para el conjunto de los países, los tres primeros lugares los ocupan los homosexuales, los ateos y los militares.

12. El conjunto de preguntas dice:

1) "De los siguientes grupos icuál es el que menos le gusta? Si hay algủn otro que le guste menos y no está en esta lista, por favor escribalo: los ateos, los homosexuales, los extremistas de derecha, los extremistas de izquierda (comunistas), los militares, gente de otra raza o de otra nacionalidad ¡cuál?, me disgusta otro grupo ¿cuál?, ningún grupo de toda la sociedad me disgus. ta".

2) "Pensando en el grupo que usted marcó como el que menos le gusta, conteste por favor las siguientes preguntas: ¿aprueba o desaprueba que personas pertenecientes al grupo que usted marcó puedan:

- enseñar en escuelas públicas

- se les pueda elegir para cargos públicos

- $\quad$ se les permita hablar en televisión o en reuniones públicas?" 


\section{Cuadro 4 \\ Distribución porcentual del grupo menos gustado por país}

\begin{tabular}{lccccc}
\hline Grupo & Guatemala & Honduras & El Salvador & Nicaragua & Costa Rica \\
\hline (N) & $(1559)$ & $(2304)$ & $(1592)$ & $(1835)$ & $(1143)$ \\
Total & 100 & 100 & 100 & 100 & 100 \\
& & & & & \\
Homosexuales & 32.8 & 35.5 & 37.2 & 28.1 & 29.0 \\
Ateos & 13.9 & 17.1 & 15.8 & 15.1 & 11.4 \\
Militares & 10.4 & 6.0 & 5.3 & 8.4 & $19.41^{13}$ \\
Ningún grupo & & & & & \\
me disgusta & 17.3 & 17.8 & 13.1 & 11.3 & 13.1 \\
Otros & 11.1 & 11.0 & 16.4 & 16.6 & 22.8 \\
Ns/Nr & 14.5 & 12.6 & 12.2 & 20.52 & 4.3 \\
\hline
\end{tabular}

Como se ve, el rechazo a los homosexuales es muy superior a la segunda opción en todos los países. También existen diferencias por edad con respecto a ese rechazo, ya que son los jóvenes de sétimo año los que rechazan con mayor fuerza a ese grupo (Cuadro 5).

Hay diferencias por género, especialmente en Guatemala, Honduras y Costa Rica (Cuadro 5), donde las mujeres son más tolerantes con los homosexuales.

\footnotetext{
13. En Costa Rica este grupo verdaderamente no existe. Sin embargo la respuesta es significativamente superior a la del resto de los países. La tradición civilista de ese país y la forma en que el antirnilitarismo y la abolición del ejército han jugaclo un papel en la construcción del orgullo nacional, explica probablemente el resuitado.
} 


\section{Cuadro 5}

Porcentaje que rechaza a los homosexuales por país según nivel de estudios y género

Nivel/Sexo Guatemala Honduras El Salvador Nicaragua Costa Rica

Nivel de estudios

\begin{tabular}{|c|c|c|c|c|c|}
\hline $\begin{array}{l}(\mathrm{NU}) \\
(\mathrm{NS})\end{array}$ & $\begin{array}{l}(754) \\
(805)\end{array}$ & $\begin{array}{l}(1099) \\
(1205)\end{array}$ & $\begin{array}{l}(809) \\
(783)\end{array}$ & $\begin{array}{l}(871) \\
(964)\end{array}$ & $\begin{array}{l}(562) \\
(581)\end{array}$ \\
\hline Undécimos & 28.6 & 26.9 & 30.2 & 21.9 & 22.4 \\
\hline $\begin{array}{l}\text { Sétimos } \\
\text { Diferencia }\end{array}$ & 36.8 & 43.2 & 44.4 & 33.7 & 35.5 \\
\hline porcentual & -8.2 & -16.3 & -14.2 & -11.8 & -13 \\
\hline
\end{tabular}

Género

\begin{tabular}{lccccc}
$(\mathrm{NH})$ & $(611)$ & $(1046)$ & $(801)$ & $(782)$ & $(509)$ \\
$(\mathrm{NM})$ & $(948)$ & $(1258)$ & $(791)$ & $(1053)$ & $(634)$ \\
& & & & & \\
Hombres & 42.2 & 46.1 & 41.7 & 33.4 & 39.9 \\
$\begin{array}{l}\text { Mujeres } \\
\text { Diferencia }\end{array}$ & 26.8 & 26.6 & 32.6 & 24.2 & 20.3 \\
porcentual & 15.4 & 19.5 & 9.1 & 9.2 & 19.6 \\
\hline
\end{tabular}

Con respecto a la aprobación o desaprobación de cada uno de los derechos para el grupo menos gustado, se pueden hacer varias observaciones. 


\section{Cuadro 6}

Porcentaje que desaprueba derechos para gente del grupo menos gustado por país

Derechos Guatemala Honduras El Salvador Nicaragua Costa Rica

$\begin{array}{lllll}(\mathrm{N}) & (1559) & (2304) & \text { (1592) }\end{array}$

Enseñar en

escuelas

68.4

69.5

71.6

57.8

74.9

Elegirse en

cargos públicos

69.0

69.4

73.6

64.2

76.0

Hablar en TV y

reuniones públicas 50.7

50.8

55.7

42.2

45.6

Llama la atención la diferencia que se da en los niveles de aprobación y desaprobación entre los dos primeros derechos y el último. Que exista mayor tolerancia para que personas del grupo menos gustado puedan hablar en televisión y en reuniones públicas puede encontrar diversas explicaciones. En primer lugar, que el derecho a la libre expresión parece tener una jerarquía especial en el sistema de valores de los jóvenes. La libertad, y la libertad de expresión en particular, ocuparon el primer lugar en las menciones de los estudiantes cuando, en una pregunta abierta, se les pidió definir la democracia.

Por otra parte, es posible sugerir la hipótesis de que la ocupación de un cargo público o de una posición docente puede ser percibida como potencialmente de mayor incidencia sobre las personas.

También existen diferencias por nivel de estudios de estos resultados, ya que los jóvenes de undécimo resultan ser más intolerantes (Cuadro 7), mostrando la relevancia de incidir durante los años de educación secundaria sobre la construcción del respeto a quienes son diferentes. 
Cuadro 7

Porcentaje que desaprueba el derecho de enseñar en escuelas públicas y ocupar cargos públicos para el grupo menos gustado por país según nivel de estudios

Nivel de estudios Guatemala Honduras El Salvador Nicaragua Costa Rica

$\begin{array}{llllll}(\mathrm{NU}) & (754) & (1099) & (809) & (871) & (562) \\ (\mathrm{NS}) & (805) & (1205) & (783) & (964) & (581)\end{array}$

Enseñar en escuelas públicas

$\begin{array}{lccccc}\text { Undécimos } & 76.9 & 77.0 & 76.4 & 65.2 & 77.9 \\ \begin{array}{l}\text { Sétimos } \\ \begin{array}{l}\text { Diferencia } \\ \text { porcentual }\end{array}\end{array} & 60.9 & 62.7 & 66.8 & 51.1 & 72.0 \\ & 16.0 & 14.3 & 9.6 & 14.1 & 5.9\end{array}$

A ocupar cargos públicos

\begin{tabular}{lccccc} 
Undécimos & 75.7 & 73.8 & 77.8 & 69.3 & 78.8 \\
Sétimos & 63.0 & 65.4 & 69.5 & 59.5 & 73.2 \\
$\begin{array}{l}\text { Diferencia } \\
\text { Porcentual }\end{array}$ & 12.7 & 8.4 & 8.3 & 9.8 & 5.6 \\
\hline
\end{tabular}

A manera de síntesis, se construyó un índice con las cuatro preguntas utilizadas. El valor del índice tiene un rango entre 0 y 10 .

En el Anexo se detallan las distribuciones del índice por país, según año de estudios que cursa y género.

La conclusión principal que se obtiene de la distribución del índice es que existe un nivel elevado de intolerancia compartido por los y las adolescentes de todos los países centroamericanos. La moda, o sea el valor del índice con una mayor frecuencia para todos los países es 0 . Allí están quienes consideran que los miembros de los grupos menos 
gustados no deben tener los tres derechos sobre los que fueron interrogados. En una democracia más profunda estos deberían ser derechos aceptados para la gran mayoría de la ciudadanía.

Dos menciones especiales deben hacerse. El Salvador es el país donde cero, el valor del índice que indica el mayor grado de intolerancia, posee una frecuencia mayor. Nicaragua es el país donde ese valor posee la menor frecuencia, pero ello puede estar afectado por la elevada no respuesta.

\section{Solidaridad}

Para medir el valor de la solidaridad se hicieron tres preguntas. La primera mide si el entrevistado percibe que la gente se preocupa más por sí misma o por ayudar a los demás. El objetivo es obtener una visión sobre la extensión del valor de la solidaridad en la sociedad ${ }^{14}$. La segunda mide la percepción sobre la importancia que el mismo entrevistado otorga a trabajar por el bienestar de los demás, con independencia de las diversas concepciones sobre cómo se debe ejercer la solidaridad ${ }^{15}$. La tercera evalúa la percepción sobre las causas de la pobreza en el país, determina el énfasis que ponen los propios estudiantes entre las responsabilidades colectivas e individuales, y permite conocer cuáles son los espacios para la generación de la demanda ciudadana por formas más estructurales para el ejercicio de la solidaridad ${ }^{16}$.

\footnotetext{
14. La pregunta dice: "Y cree usted que la mayoría de las veces la gente se preocupa solo por sí misına, o cree yue la mayoríd de las veces la gente trata de ayudar la prójimo?" 15. La pregunta dice: "Hay principios o valores que le sirven a las personas de guía para actuar en la vida. En su caso particular, usted diría que trabajar por el bienestar de otros sin esperai nada a cambio es para usted...?"

Sigue una opción de cinco posiciones con "nada importante" y "muy importante" en los extremos.

16. La pregunta dice: "¿Por qué cree usted que hay pobreza en nuestro país? Porque...". Sigue una opción de cinco posiciones con "las personas son vagas" y "la sociedad los trata injustamente" en los extremos.
} 
Se buscó utilizar preguntas que midieran el valor de la solidaridad con independencia de las diferencias ideológicas que puedan existir, relativas al modo en que ésta se debe concretar en acciones. Se evalúa la percepción de la solidaridad colectiva, la disposición declarada hacia actos solidarios y la responsabilidad colectiva ante la pobreza.

Para obtener las respuestas se ofreció una escala de cinco posiciones en cada una de las preguntas. Para este artículo, sin embargo, se redujo la escala a tres posiciones.

El siguiente cuadro muestra los resultados para la primer pregunta:

\section{Cuadro 8}

La gente se preocupa más por sí misma o por ayudar al prójimo por país según nivel de estudios

Nivel de

estudios

Guatemala Honduras El Salvador Nicaragua Costa Rica

(N)

(1559)

(2304)

(1592)

(1835)

(1143)

Por sí misma

$\begin{array}{llllll}\text { Undécimos } & 67.5 & 72.9 & 70.3 & 67.8 & 69.0 \\ \begin{array}{l}\text { Sétimos } \\ \begin{array}{l}\text { Diferencia } \\ \text { porcentual }\end{array}\end{array} & 53.4 & 48.2 & 46.8 & 43.0 & 52.0 \\ & 14.1 & 24.7 & 23.5 & 24.8 & 17.0\end{array}$

Por ayudar al prójimo

\begin{tabular}{lrrrrr} 
Undécimos & 8.8 & 9.9 & 10.1 & 13.0 & 7.2 \\
$\begin{array}{l}\text { Sérimos } \\
\text { Diferencia }\end{array}$ & 18.9 & 29.8 & 28.3 & 40.7 & 18.6 \\
Porcentual & -10.1 & -19.9 & -18.2 & -27.7 & -11.4 \\
\hline
\end{tabular}


Los jóvenes se inclinan por afirmar que las personas se preocupan más por sí mismas que por los demás. Son mucho más los jóvenes de undécimo año que consideran que las personas actúan de manera más egoísta y menos generosa con los demás. Los contrastes entre sétimo y undécimo son especialmente pronunciados en Honduras, El Salvador $y$, principalmente, en Nicaragua.

Por qué ocurre este cambio sobre la percepción de la solidaridad de los otros, es una interrogante relevante para investigaciones posteriores.

Tanto en los hombres como en las mujeres más de la mitad opina que la gente se preocupa más por sí misma. Es relevante la diferencia de las mujeres en Guatemala, Honduras y El Salvador donde esta percepción es aún más pronunciada (Cuadro 9).

\section{Cuadro 9}

Porcentaje que respondió afirmativamente que la gente se preocupa más por sí misma por país según sexo

Sexo

Guatemala Honduras El Salvador Nicaragua Costa Rica

\begin{tabular}{lccccc}
\hline (NM) & $(611)$ & $(1046)$ & $(801)$ & $(782)$ & $(509)$ \\
$(\mathrm{NH})$ & $(948)$ & $(1258)$ & $(791)$ & $(1053)$ & $(634)$ \\
& & & & & \\
Mujeres & 63.9 & 64.6 & 63.6 & 56.3 & 60.1 \\
Hombres & 54.5 & 54.5 & 54.1 & 52.8 & 60.7 \\
$\begin{array}{l}\text { Diferencia } \\
\text { Porcentual }\end{array}$ & 9.4 & 10.1 & 9.5 & 3.5 & -0.6 \\
\hline
\end{tabular}

La pregunta sobre la importancia que se concede a trabajar por el bienestar de otros sin esperar nada a cambio (Cuadro 10) mide la convicción altruista de que es importante para cada individuo trabajar para el beneficio social. 
Quizás el único interés que podría estar detrás de esta convicción es que solo mediante el bienestar colectivo es posible alcanzar el bienestar individual.

Cuadro 10

Importancia de trabajar por el bienestar de otros por país según nivel de estudios (en porcentajes)

\begin{tabular}{|c|c|c|c|c|c|}
\hline $\begin{array}{l}\text { Nivel de } \\
\text { estadios }\end{array}$ & Guatemala & Honduras & El Salvador & Nicaragua & Costa Rica \\
\hline Total & 100 & 100 & 100 & 100 & 100 \\
\hline (N) & (754) & (1099) & (809) & (871) & (562) \\
\hline \multicolumn{6}{|l|}{ Undécimos } \\
\hline $\begin{array}{l}\text { No es } \\
\text { importante } \\
\text { Posición }\end{array}$ & 12.2 & 14.9 & 13.2 & 23.6 & 8.5 \\
\hline & 24.5 & 17.7 & 17.3 & 21.2 & 21.5 \\
\hline importante & 60.7 & 63.9 & 65.8 & 49.0 & 68.7 \\
\hline $\mathrm{Ns}_{\mathrm{s}} / \mathrm{Nr}$ & 2.5 & 3.5 & 3.7 & 6.2 & 1.2 \\
\hline$(\mathrm{N})$ & (805) & (1205) & (783) & (964) & (581) \\
\hline \multicolumn{6}{|l|}{ Sétimos } \\
\hline $\begin{array}{l}\text { No es } \\
\text { importante } \\
\text { Posición }\end{array}$ & 20.2 & 26.3 & 18.2 & 31.9 & 13.0 \\
\hline $\begin{array}{l}\text { intermedia } \\
\text { Sí es }\end{array}$ & 19.6 & 15.0 & 15.1 & 16.7 & 25.6 \\
\hline importante & 53.6 & 52.7 & 60.0 & 45.0 & 57.2 \\
\hline $\mathrm{Ns} / \mathrm{Nr}$ & 6.6 & 5.9 & 6.6 & 6.3 & 4.1 \\
\hline
\end{tabular}


Las respuestas muestran un predominio de la valoración de las prácticas solidarias, especialmente en undécimo. Esta valoración es mayor en Costa Rica, e intermedia en El Salvador, Honduras y Guatemala.

Nicaragua es el país cuya manifestación de la solidaridad es menor, ya que alrededor de una cuarta parte de sus jóvenes de undécimo dice que trabajar por el bienestar de otros es poco o nada importante, y alrededor de un tercio de los de sétimo opina también de la misma forma.

La tercera pregunta que se realizó para medir el valor de la solidaridad es la que busca identificar las causas de la pobreza en el país, estando en un extremo la opción de que la pobreza se debe a que las personas son vagas y, en el otro la opción de que la sociedad injusta es la responsable de esa situación (Cuadro 11).

\section{Cuadro 11}

Distribución porcentual de las respuestas a la pregunta ¿por qué hay pobreza en nuestro país? por país

Causa

Guatemala Honduras El Salvador Nicaragua Costa Rica

Total

100

100

100

100

100

(N)

(1559)

(2304)

(1592)

(1835)

Sociedad injusta

43.3

38.3

48.2

56.0

23.0

Posición

intermedia

27.2

22.1

23.1

16.6

39.3

Personas vagas

23.5

32.2

18.7

17.2

33.4

$\mathrm{Ns} / \mathrm{Nr}$

6.0

7.4

9.9

10.1

4.4

Las respuestas obtenidas muestran una mayor concentración de personas que señalan que la pobreza se debe a 
que la sociedad trata de manera injusta a las personas. Esta afirmación es particularmente fuerte en el caso de Nicaragua.

Contrasta esto con las respuestas de Costa Rica, país en el que un porcentaje mayor se coloca en la posición intermedia y en el que el peso mayor entre los extremos no recae sobre la injusticia social. Esto se puede deber a que la inversión social que Costa Rica ha hecho por años -y que se refleja en diversos indicadores sociales- ha generado un clima de bienestar suficiente como para no culpar tanto a la sociedad por este problema.

Por otra parte, tanto en Costa Rica como en Honduras una tercera parte de los jóvenes afirma que la pobreza existe porque las personas son vagas.

También hay que decir que son más los jóvenes de sétimo año de Nicaragua y El Salvador que afirman que la responsabilidad de la pobreza es de la sociedad injusta, tal y como se explicita en el Cuadro 12.

\section{Cuadro 12}

Porcentaje que afirma que hay pobreza porque la sociedad trata a las personas injustamente por país según nivel de estudios

Nivel de estudios Guatemala Honduras El Salvador Nicaragua Costa Rica

\begin{tabular}{lccccc}
\hline$(\mathrm{N})$ & $(1559)$ & $(2304)$ & $(1592)$ & $(1835)$ & $(1143)$ \\
Undécimos & 41.2 & 35.0 & 42.5 & 49.2 & 13.0 \\
$\begin{array}{l}\text { Sétimos } \\
\begin{array}{l}\text { Diferencia } \\
\text { porcentual }\end{array}\end{array}$ & 45.3 & 41.4 & 54.2 & 62.2 & 32.7 \\
\hline
\end{tabular}

Las únicas diferencias por género relevantes se encuentran en Nicaragua donde más mujeres $(61.8 \%)$ que hombres 
(48.2\%) expresan que la causa de la pobreza son las injusticias de la sociedad, y más hombres $(22.5 \%)$ que mujeres $(13.3 \%)$ opinan que la pobreza es debida a que las personas son vagas.

Estas tres preguntas sobre solidaridad, permiten concluir que existe en el discurso de los estudiantes una valoración elevada de los valores altruistas, a los que, según su percepción, la sociedad no les da igual importancia.

\section{Conclusiones}

Existe en la población estudiada un valor bastante cimentado de igualdad política. No obstante, es importante no subestimar al grupo que está dispuesto a renunciar al principio de la igualdad cuando se le anteponen argumentos antidemocráticos y cuya extensión en algunos países es de hasta un $25 \%$.

La intolerancia hacia la diversidad es un problema generalizado. Una mayoría de los jóvenes niega el derecho a enseñar, a ser electo en cargos públicos y, en menor grado, a expresarse libremente a personas del grupo menos gustado. En todos los países, el valor del índice más frecuente es el que lè niega todos los derechos al grupo menos gustado. Ello es aún más fuerte en El Salvador.

Por otra parte, es muy grave que los resultados sugieran que la intolerancia crece durante los años que separan a los sétimos años de los undécimos. Esto ocurre especialmente en Guatemala, Honduras y Nicaragua.

Este problema de intolerancia amenaza la calidad de las' democracias centroamericanas, pues puede conducir a la aceptación de violaciones de los derechos fundamentales de otros. La justificación del atropello a los derechos de las personas ha sido un elemento constitutivo de los regímenes autoritarios, sea por disidencia política o por estigmatización social. 
La solidaridad es un valor extendido, especialmente en undécimo año, aunque con variaciones por país. Nicaragua es donde la manifestación del altruismo está más disminuida. La percepción de cuán solidaria es la sociedad muestra un mayor pesimismo en los de undécimo año. Estos mismos son los que afirman con mayor vehemencia el valor de trabajar por el bienestar de los demás.

Así, entre sétimos y undécimos hay una brecha con respecto a la solidaridad, ya que disminuye la percepción de que las personas son generosas y se preocupan por ayudar a los demás. Sin embargo, crece la convicción personal de que es importante trabajar por el bienestar de los otros sin esperar nada a cambio.

La mayor parte de los estudiantes piensa que la pobreza se debe a que la sociedad trata de manera injusta a las personas y no a que las personas son vagas. Esto sucede muy especialmente en Nicaragua, y Costa Rica es la excepción.

Dadas las fortalezas y las debilidades encontradas en los valores de las y los jóvenes centroamericanos, es imperioso profundizar en el conocimiento sobre los mecanismos de transmisión y fortalecimiento de los valores democráticos y diseñar acciones que vayan en esa dirección. Esto es especialmente relevante en el caso de la tolerancia hacia la diversidad.

Del lado positivo, hay importantes oportunidades para el mayor desarrollo de la cultura democrática: el valor de la igualdad política tiene cimientos importantes, y hay un principio altruista que es muy valorado por los jóvenes, que además crece durante el período en que transcurre la educación secundaria.

Actuar enfrentando los problemas, con el conocimiento de las oportunidades existentes, es de vital importancia para afirmar positivamente este componente crítico de la cultura política de la región e intentar con ello mejorar la 
calidad de nuestras democracias y avanzar en el camino de su sostenibilidad y profundización.

\section{Bibliografía}

Booth, John A. y Mitchell Seligson (1993). "Paths to Democracy and the Political Culture of Costa Rica, Mexico and Nicaragua" en Political Culture and Democracy in Developing Countries, Larry Diamond (editor), Lynne Rienner Publishers, Colorado.

Lipset, Seymour M. (1963). El hombre político, Editorial Universitaria de Buenos Aires.

Putnam, Robert D. (1993). Making Democracy Work: Civic Traditions in Modern Italy, Princeton University Press.

Rodríguez, Florisabel, Silvia Castro Méndez y Guillermo Monge Guevara (2002) "Cultura política: orientaciones conceptuales", en Serie de Cuadernos de Trabajo, no. 02, Procesos: San José.

Tocqueville, Alexis de (1969). Democracy in America, Anchor Books, New York.

Waltzer, Michael (1997). On Toleration, New Haven: Yale University Press. 


\section{Anexo}

\section{Índice de tolerancia por país controlado por nivel de estudios y género}

\begin{tabular}{|c|c|c|c|c|c|c|c|c|c|c|c|c|c|c|c|c|c|c|c|c|c|c|c|c|c|}
\hline \multirow{4}{*}{$\begin{array}{l}\text { hidade } \\
\text { Tolderancia" }\end{array}$} & \multicolumn{25}{|c|}{$P_{25}$} \\
\hline & \multicolumn{5}{|c|}{ Gutemath } & \multicolumn{5}{|c|}{ Hendras } & \multicolumn{5}{|c|}{ ElSavador } & \multicolumn{5}{|c|}{ Nicaraga } & \multicolumn{5}{|c|}{ CostaRica } \\
\hline & \multirow[t]{2}{*}{ Tad } & \multicolumn{2}{|c|}{$\begin{array}{l}\text { Abou } \\
\text { anu }\end{array}$} & \multicolumn{2}{|c|}{ Getrero } & \multirow[t]{2}{*}{ Tal } & \multicolumn{2}{|c|}{$\begin{array}{l}\text { Ainque } \\
\text { arsa }\end{array}$} & \multicolumn{2}{|c|}{ Genero } & \multirow[t]{2}{*}{ Tdal } & \multicolumn{2}{|c|}{$\begin{array}{l}\text { Aioque } \\
\text { carsa }\end{array}$} & \multicolumn{2}{|c|}{ Ginero } & \multirow[t]{2}{*}{ Totad } & \multicolumn{2}{|c|}{$\begin{array}{c}\text { Ainque } \\
\text { arsa }\end{array}$} & \multicolumn{2}{|c|}{ Género } & \multirow[t]{2}{*}{ Todal } & \multicolumn{2}{|c|}{$\begin{array}{c}\text { Anoque } \\
\text { cursa }\end{array}$} & \multicolumn{2}{|c|}{ Género } \\
\hline & & $r$ & $11^{\circ}$ & Masc & Fom & & $r$ & $11^{\prime}$ & Masc & Fem & & $r$ & $11^{\circ}$ & Masc & Fem & & $r$ & $11^{\circ}$ & Masc & Fem & & $r$ & 110 & Masc & Fen \\
\hline Tdal & 100 & 100 & 120 & 100 & 100 & 100 & 100 & 100 & 100 & 100 & 100 & 100 & 100 & 100 & 100 & 100 & 100 & 100 & 100 & 100 & 100 & no & 100 & 100 & 100 \\
\hline (N) & (1989) & (201) & (15A) & (611) & (948) & (2004) & (1205) & (1009) & (1066) & (1258) & (1592) & $(123)$ & $(209)$ & $(801)$ & $(P 91)$ & (1825) & (964) & (871) & (782) & (1063) & $(1143)$ & (581) & 52 & $(50)$ & (634) \\
\hline 0 & 37,1 & 36,1 & 362 & $38 \beta$ & 360 & 36,6 & 352 & 380 & 392 & 34,5 & 462 & 46,5 & 45,8 & 47,8 & 44,4 & 225 & 29,0 & 35,8 & 34,4 & 312 & 389 & 38,3 & 39,4 & 41,8 & 36,4 \\
\hline 3 & 212 & 216 & 208 & 235 & $19 \beta$ & 202 & 213 & 19,0 & 222 & 18,5 & 192 & 19,5 & 19,0 & 185 & 199 & 21,1 & 21,8 & 20,5 & 209 & 21,4 & 26,9 & 283 & 25,5 & 25,5 & 28,0 \\
\hline 7 & 10,7 & 13,5 & 8.1 & 135 & 89 & 122 & 15,1 & 9,5 & 140 & 10,8 & 10,1 & 120 & 84 & 10,7 & 9,3 & 179 & 23,4 & 12,7 & 21,1 & 15,6 & 11,7 & 12,1 & 11,3 & 11,5 & 11,9 \\
\hline 10 & 30,9 & 2888 & 329 & 242 & 353 & 31,0 & 28,4 & 33.5 & 24,5 & 36,3 & 246 & 220 & 26,9 & 229 & 26,4 & 28,4 & 25,7 & 310 & 23,7 & 319 & 226 & 21,3 & 238 & 212 & 23,7 \\
\hline \multicolumn{26}{|c|}{ Estadisicos deresumen } \\
\hline Promedio & 4,48 & 4,48 & 4,48 & 4,07 & 4,75 & 456 & 4,54 & 458 & 4,10 & 494 & 3,74 & 352 & 384 & 3,00 & $3 \not 89$ & 4,73 & 4,86 & 4,60 & 4,47 & $4 M 2$ & 3,88 & 3,83 & 394 & 360 & 4,04 \\
\hline Moda & 0 & 0 & 0 & 0 & 0 & 0 & 0 & 0 & 0 & 10 & 0 & 0 & 0 & 0 & 0 & 0 & 0 & 0 & 0 & 10 & 0 & 0 & 0 & 0 & 0 \\
\hline Medana & 3 & 3 & 3 & 3 & 3 & 3 & 3 & 3 & 3 & 3 & 3 & 3 & 3 & 3 & 3 & 3 & 3 & 3 & 3 & 3 & 3 & 3 & 3 & 3 & 3 \\
\hline
\end{tabular}

